\title{
ENFERMERÍA EN EL TRATAMIENTO DE LA FOBIA A TRAGAR EN LA INFANCIA Y LA ADOLESCENCIA: UNA REVISIÓN BIBLIOGRÁFICA
}

\section{JORDI TORRALBAS-ORTEGA ${ }^{1}$, JOAQUIM PUNTÍ-VIDAL', VICTORIA VALLS-IBÁÑEZ², ELOÍSA ARIAS-NÚÑEZ1', MARI CARMEN NARANJO-DÍAZ1 , IGOR MERODIO-RUIZ1 Y ANTONIA BRETONES-RODRÍGUEZ ${ }^{3}$}

${ }^{1}$ Centro de Salud Mental Infanto-Juvenil. Salut Mental Parc Taulí. Corporació Sanitària Parc Taulí. Institut Universitari Parc Taulí-UAB. Sabadell (Barcelona).

${ }^{2}$ Centro de Atención Primaria La Serra. Institut Català de la Salut. Sabadell (Barcelona).

${ }^{3}$ Centre de Salut Mental Infantil i Juvenil de I'Eixample. Institut Clínic de Neurociències.

Hospital Clínic de Barcelona.

\section{RESUMEN}

Introducción: la fobia a la deglución se caracteriza por el miedo a atragantarse al ingerir comida, líquidos o pastillas. Aunque es un trastorno considerado menor y de baja prevalencia dentro de los trastornos alimentarios de la infancia y la adolescencia, en estos últimos años, se ha observado un aumento de su incidencia en la etapa infantil y juvenil.

Método: se han analizado las publicaciones de los últimos 20 años (1995-2015), en las que se recogen un total de 42 casos con fobia a tragar, recopilándose las principales variables clínicas y terapéuticas.

Resultados: este trastorno parece ser más común entre el sexo femenino en la etapa infantil, con elevada comorbilidad con otros trastornos, especialmente, de ansiedad. Existen antecedentes traumáticos en un $57,1 \%$ de la muestra, y se han mostrado eficaces distintos tratamientos basados en la terapia cognitivo-conductual y el tratamiento farmacológico, con recuperación completa en un 80 \% de los casos. La aportación de la enfermería al plan terapéutico no se menciona en la mayoría de las publicaciones.

Conclusiones: es indispensable la inclusión del trastorno en el diagnóstico diferencial de los trastornos de la alimentación en la infancia, y su conocimiento por parte de las enfermeras de atención primaria, con la finalidad de identificarlo específicamente y poder establecer un adecuado tratamiento multidisciplinario precoz que mejore los resultados terapéuticos.

Palabras clave: fobia, deglución, tragar, atragantarse, infancia, adolescencia, tratamiento. 


\section{INTRODUCCIÓN}

Los problemas alimentarios en la niñez y la adolescencia son cada vez más reconocidos, especialmente, los trastornos de la conducta alimentaria (TCA) $y$, en particular, la anorexia nerviosa (AN) ${ }^{1}$.

Otros problemas de características similares, con consecuencias de igual magnitud tanto a nivel orgánico como psicológico, no son considerados habitualmente en el diagnóstico diferencial. Este es el caso de la fobia a tragar o atragantarse, cuya primera descripción clínica aparece en $1978^{2}$, y que se caracteriza por el miedo excesivo e irracional a atragantarse al ingerir comida, líquidos o pastillas.

En su sentido más amplio, las fobias han sido estudiadas desde modelos psicológicos muy diversos, tanto comprehensivos como intervencionistas. La prevalencia de las fobias es variable según el tipo de miedo estudiado. En el caso de la fobia a la deglución, su prevalencia e incidencia por sexo es desconocida ${ }^{3}$; sin embargo, se ha constatado un aumento considerable de su descripción en diversos artículos de revisión o de casos clínicos de los últimos cinco años. Okada et al. ${ }^{4}$ sitúan la incidencia en la muestra clínica en el 1,9\% en un período de estudio de cinco años, en los pacientes atendidos en consultas externas de salud mental.

Por todo ello, no resulta extraño que la fobia a tragar no sea reconocida como una entidad nosológica propia en las principales clasificaciones internacionales; así, la cuarta edición del Manual diagnóstico y estadístico de los trastornos mentales (DSM-IV-TR ${ }^{5}$ ) la incluye como una fobia específica dentro de su categoría residual, junto con la fobia a vomitar o a contraer una enfermedad. En la reciente versión del $D S M-V^{6}$, aparece en la categoría de trastorno de la alimentación por evitación/restricción.

Finalmente, en relación con su comorbilidad, este trastorno se asocia habitualmente a complicaciones

Correspondencia: J. Torralbas Ortega. Correo electrónico: hdatauli@live.com físicas como la deshidratación, la desnutrición y la pérdida de peso, y a problemas psiquiátricos derivados de sus actitudes evitativas y relacionados con otros trastornos psicopatológicos del espectro ansioso.

La finalidad de este artículo de revisión es determinar las principales características clínicas y terapéuticas de la fobia a la deglución, en el caso específico de niños y adolescentes, y establecer el papel que desempeñan los cuidados de enfermería en ellos.

\section{METODOLOGÍA}

Se han analizado las publicaciones recogidas en PsycINFO, PubMed y Google Scholar en los últimos 20 años, con la finalidad de poder recoger el máximo de aportaciones científicas en este campo, desde la visión de cualquier disciplina.

Las palabras clave utilizadas en español fueron: «fobia», «deglución» o «tragar» o «atragantarse», «infancia»y «adolescencia». En inglés, se utilizaron las key words "phobia», "choking» 0 "swallowing», y "phagophobia», con los limitadores de edad "childhood» $\mathrm{y}$ «adolescence».

En PsycINFO y PubMed, se recogieron un total de 17 reseñas bibliográficas. Dos de ellas se excluyeron: una por tratarse de un artículo teórico sobre el problema y otra por ser un libro editado que simplemente citaba el trastorno en uno de sus capítuIos. En Google Scholar, se encontraron dos artículos distintos a los anteriores, publicados en revistas españolas no indexadas en PsyclNFO y PubMed.

Dos artículos publicados por los mismos autores (de Lucas-Taracena y Montañés-Rada, 2005 y 2006) son dos manuscritos de revisión de los años 1978 a 2005, con el mismo contenido de sujetos. Se excluyó el artículo que hacía referencia a la población adulta, recuperando el artículo de revisión referente a la población infantil y adolescente. Los datos de los estudios incluidos en la revisión, anteriores a 1995, fueron analizados junto al resto de artículos recuperados desde esta fecha, ya que algunos no habían sido incluidos en la revisión realizada por de Lucas 
y Montañés en 2005, debido a los criterios de inclusión que los autores habían utilizado.

Así, un total de 16 artículos fueron analizados en este estudio de revisión. De cada uno de ellos, se recogieron las variables: número de casos analizados, edad expresada en años cumplidos, sexo, comorbilidad con otros trastornos, tiempo de evolución hasta el tratamiento, tipo de tratamiento empleado, evolución en el seguimiento del caso (recuperación completa, parcial, transformación del trastorno o recidiva), presencia de antecedentes traumáticos y pérdida de peso, siempre que estos datos estuvieran disponibles en los diferentes estudios (tabla 1).

\section{RESULTADOS}

Fueron analizados un total de 42 casos de niños y adolescentes en los 16 artículos, con edades comprendidas entre los 3 y los 15 años (media: 9,4 \pm 3,2 años). Un $69 \%$ de la muestra era de sexo femenino.

El $57,1 \%$ de los casos se inicia con un suceso traumático de atragantamiento (frutos secos, monedas, anillo, pan, etc.), en general, de rápida solución, no requiriendo asistencia médica especializada. En el resto de casos, se inicia a partir de la visualización de un episodio de atragantamiento en otra persona (normalmente, familiar) o por un comentario sobre un proceso de asfixia al tragar, o tras dolor faríngeo secundario a una enfermedad médica que le imposibilitaba la ingesta normal de alimentos. Solo cuatro casos de los 32 informados no presentaron ningún factor estresante aparente anterior al trastorno.

\section{Comorbilidad del trastorno}

Se ha asociado a elevada comorbilidad, sobre todo, con trastornos de ansiedad en la infancia ${ }^{3,7}$. De la muestra recogida, en el 72,7\% de los casos, existe un diagnóstico asociado a algún tipo de trastorno ansioso (trastorno de pánico, obsesivo-compulsivo [TOC], de ansiedad por separación, de personali-
La fobia a tragar o atragantarse se caracteriza por el miedo excesivo e irracional a atragantarse al ingerir comida, líquidos o pastillas

dad evitativa-ansiosa, etc.). Un caso se identifica como comórbido con un trastorno por déficit de atención e hiperactividad, en un adolescente de 13 años, que recibe tratamiento con metilfenidato, sin mencionar pérdida de apetito ${ }^{8}$. Solamente en 23 de los 42 sujetos incluidos en el estudio se había informado de los diagnósticos comórbidos. En algunos artículos, se menciona el tipo de interacción familiar ${ }^{9}$, el carácter premórbido del niño $0^{4}$ o posibles factores estresantes ambientales ${ }^{10}$, pero no se especifican otros problemas asociados a la fobia a tragar.

\section{Diagnóstico diferencial}

Varios autores plantean la necesidad de diagnóstico diferencial con distintos trastornos $7,8,11-14$, tanto psicológicos como orgánicos.

Se considera indispensable un primer cribado a nivel fisiológico que descarte toda enfermedad orgánica que pueda dificultar la deglución (examen peribucal y orofaríngeo, esofagograma o estudio modificado de la deglución de bario) por parte de un especialista.

Para el establecimiento del diagnóstico diferencial con otros problemas, se define el miedo a atragantarse como fundamental para distinguir este trastorno del reflejo nauseoso hipersensible, la disfagia o el globo histérico, donde este temor fóbico no se presenta; el rechazo al alimento frente al miedo a tragar, en el caso de la AN o la fobia a la comida; el miedo a ahogarse durante una crisis de pánico y a comer en presencia de otros, en el caso del trastorno de pánico y en el caso de la fobia social, respectivamente; la sintomatología obsesiva propia del TOC, donde existen pensamientos claramente intrusivos, que, habitualmente, se acompañan de rituales o conductas 
Tabla 1. Casos publicados de fobia a tragar (1988-2015)

\begin{tabular}{|c|c|c|c|c|c|c|c|c|c|}
\hline Referencia & Casos & Edades & Sexo & Comorbilidad & Tiempo de evolución & Tratamiento & Evolución & Antecedentes traumáticos & Pérdida de peso \\
\hline $\begin{array}{l}\text { Chatoor et al., } 1988 \\
\text { (Procede de: de Lucas y Montañés, 2005) }\end{array}$ & 5 & 8-11 años & $\begin{array}{l}2 \text { masculino } \\
3 \text { femenino }\end{array}$ & $\begin{array}{l}\text { TAS, DEP, } \\
\text { ND, TOC, } \\
\text { Tr. del sueño }\end{array}$ & & $\begin{array}{l}5 \text { TCC } \\
2 \text { imipramina }\end{array}$ & $\begin{array}{l}3 \mathrm{RC} \\
1 \mathrm{RP} \\
1 \mathrm{TRF}\end{array}$ & & NC \\
\hline Chorpita et al., 1997 & 1 & 13 años & Femenino & Tr. de pánico & $\sim 6$ meses & TCC & $\mathrm{RP}$ & Atrag. 5 años antes & No \\
\hline de Lucas et al., 2001 & 1 & 14 años & Femenino & & $\sim 7$ meses & TCC & $\mathrm{RC}$ & Mononucleosis & No \\
\hline Zelikovsky et al., 2001 & 1 & 5 años & Masculino & & 2 meses & TCC & $\mathrm{RP}$ & Atrag. con una moneda & NC \\
\hline Gómez y del Barrio, 2002 & 1 & 6 años & Femenino & & 2 meses & TCC & $\mathrm{RC}$ & Atrag. 1,5 meses antes & $4 \mathrm{~kg}$ \\
\hline Bailly et al., 2003 & 1 & 11 años & Femenino & TOC, TAS & 1 año & $T C C+F L X$ & $\mathrm{RP}$ & Atrag. del padre & $7 \mathrm{~kg} ;$ IMC: $14,7 \mathrm{~kg} / \mathrm{m}^{2}$ \\
\hline Burklow y Linscheid, 2004 & 6 & 5-12 años & $\begin{array}{l}1 \text { masculino } \\
5 \text { femenino }\end{array}$ & & 1-4 meses & TCC & $6 \mathrm{RC}$ & $\begin{array}{l}4 \text { atrag. } \\
1 \text { dolor de faringe } \\
1 \text { atrag. de una hermana }\end{array}$ & $3-7,5 \mathrm{~kg}$ \\
\hline Banerjee et al., 2005 & 3 & 7-12 años & $\begin{array}{l}1 \text { masculino } \\
2 \text { femenino }\end{array}$ & $\begin{array}{l}\text { Tr. de ansiedad } \\
\text { TOC }\end{array}$ & 1 mes-3,5 años & ISRS & $\mathrm{RC}$ & $\begin{array}{l}1 \text { atrag. } \\
1 \text { vómito }\end{array}$ & $0-3 \mathrm{~kg}$ \\
\hline Geffken et al., 2005 & 1 & 4 años & Masculino & TOC & 2 meses & TCC & $\mathrm{RC}$ & Atrag. con un sándwich & NC \\
\hline Montañés et al., 2005 & 4 & 9-15 años & $\begin{array}{l}2 \text { masculino } \\
2 \text { femenino }\end{array}$ & $\begin{array}{l}\text { Tr. de pánico, } \\
\text { TAS } \\
\text { PEA }\end{array}$ & 4-12 meses & $\begin{array}{l}4 \mathrm{TCC} \\
1+\mathrm{PRX}+\mathrm{LRZ} \\
1+\mathrm{CMI}+\mathrm{ALP}\end{array}$ & $\begin{array}{l}1 \text { TRF } \\
1 \text { pérdida } \\
1 \text { RP } 1 \text { RR }\end{array}$ & $\begin{array}{l}3 \text { no } \\
1 \text { atrag. con un pistacho }\end{array}$ & NC \\
\hline Çiyiltepe y Türkbay, 2006 & 1 & 13 años & 1 masculino & $\begin{array}{l}\text { TDAH } \\
\text { Tr. de lectura }\end{array}$ & 3 meses & TCC & $\mathrm{RC}$ & $\begin{array}{l}\text { Atrag. } 1 \text { año antes } \\
\text { Atrag. de la madre } 3 \text { meses antes }\end{array}$ & $4 \mathrm{~kg}$ \\
\hline Okada et al., 2007 & 6 & 5-15 años & $\begin{array}{l}1 \text { masculino } \\
5 \text { femenino }\end{array}$ & TOC, TAS & 4-24 meses & TCC & $\begin{array}{l}5 \mathrm{RC} \\
1 \mathrm{RP}\end{array}$ & $\begin{array}{l}5 \text { atrag. } \\
1 \text { enfermedad }\end{array}$ & $2-11 \mathrm{~kg}$ \\
\hline de Roos y de Jongh, 2008 & 4 & 3-15 años & $\begin{array}{l}1 \text { masculino } \\
3 \text { femenino }\end{array}$ & No & 3 semamas- 5 años & EMDR & $\mathrm{RC}$ & $\begin{array}{l}1 \text { atrag. de un amigo } \\
3 \text { atrag. }\end{array}$ & NC \\
\hline García et al., 2010 & 2 & 5-8 años & 2 femenino & & 1 mes-1 año & $\begin{array}{l}\text { TCC } \\
1+B Z D\end{array}$ & $\mathrm{RC}$ & $\begin{array}{l}1 \text { atrag. } \\
1 \text { comentario }\end{array}$ & NC \\
\hline Torralbas et al., 2012 & 4 & 8-12 años & $\begin{array}{l}3 \text { masculino } \\
1 \text { femenino }\end{array}$ & & 3-12 meses & TCC & $\mathrm{RC}$ & $\begin{array}{l}2 \text { atrag. } \\
1 \text { vómito }\end{array}$ & $8-13 \mathrm{~kg}$ \\
\hline Tanıdır y Hergüner, 2015 & 1 & 10 años & Femenino & TOC, TAS & 6 años & Mirtazapina & $\mathrm{RC}$ & Atrag. con un anillo & P10 IMC \\
\hline
\end{tabular}

ALP: alprazolam; atrag.: atragantamiento; BZD: benzodiacepinas; CMI: clomipramina; DEP: depresión; EMDR: desensibilización y

eprocesamiento por movimientos oculares (eye movement desensitization and reprocessing); $F$ LX: fluoxetina; IMC: índice de mas
corporal: ISRS: inhibidores selectivos de la recaptación de serotonina; LRZ: lorazepam: NC. no consta; ND. trastorno negativista

desafiante; P: percentil; PEA: personalidad evitativa-ansiosa; PRX: paroxetina; RC: recuperación completa; RP: recuperación parcial;

RR: recuperación con recidiva; TAS: trastorno de ansiedad por separación; TCC: terapia cognitivo-conductual; TDAH: trastorno por
déficit de atención e hiperactividad; TOC: trastorno obsesivo-compulsivo; Tr: trastorno; TRF: transformación del problema. 
de neutralización, que no se observan comúnmente en la fobia a la deglución; y en el trastorno por estrés postraumático, donde existe una reexperimentación persistente del acontecimiento traumático, aspecto que no se presenta en las fobias específicas.

\section{Tiempo de evolución del trastorno}

Aunque, en general, el problema es consultado a un especialista de forma más temprana que en la población adulta14, el tiempo medio de evolución hasta la solicitud de tratamiento en niños y adolescentes es de 7,9 meses (desviación estándar [DE]: 12,1 ), con un rango que oscila desde los 30 días hasta los 6 años, calculados en los 37 casos en los que se dispone de estos datos. En los casos de larga evolución, el problema se presentó de forma insidiosa, con adopción progresiva de restricción de alimentos hasta conseguir la anulación de cualquier ingesta sólida o semilíquida.

Aunque, en la mayoría de publicaciones, se cita la pérdida de peso como uno de los problemas físicos más frecuentes derivados de la fobia específica, solamente el 59,5\% de los autores informan de la posible pérdida de peso, aunque sí en todos se cita el aumento de peso en la recuperación del trastorno. En los cuatro casos en los que no existe esta disminución ponderal, la alimentación se centra en productos de ingesta y deglución sencillas, básicamente, carbohidratos. En el resto de niños estudiados, se anotan pérdidas ponderales importantes que van de los 2 a los $13 \mathrm{~kg}$, sin tener en cuenta la repercusión en el desarrollo pondoestatural del niño o adolescente durante el tiempo de evolución de la enfermedad.

El papel que la enfermera de los equipos de salud mental infantojuvenil puede desempeñar no se refleja en la mayoría de publicaciones

\section{Tratamiento del trastorno}

Los tratamientos empleados en la mayoría de estudios analizados se centran, básicamente, en tres pilares: terapia cognitivo-conductual (TCC), tratamiento farmacológico, y la combinación de ambos.

La TCC se ha utilizado por diversos autores de forma aislada o combinada con fármacos 4,7,8,10-19. Su aplicación es variada en cuanto a técnicas empleadas, pero, fundamentalmente, se centra en:

a) Psicoeducación sobre la fisiología de la deglución, adversidades que pueden ocurrir durante la comida y mecanismos de defensa del organismo.

b) Inhibición de la ansiedad con técnicas de control de la activación, como relajación muscular progresiva de Jacobson y ejercicios respiratorios.

c) Psicoeducación familiar sobre pautas básicas de manejo de la conducta (extinción, refuerzo positivo, etc.) y prevención de temores, establecimiento de un sistema de reforzadores estandarizado junto a la familia, autorregistros de conducta y registros alimentarios.

d) Exposición en imaginación y en vivo, con modelado simbólico y participante, juego simbólico, aproximación gradual a los estímulos temidos con refuerzo contingente a cada uno de sus progresos, moldeamiento con práctica reforzada, autoinstrucciones para el afrontamiento e imágenes emotivas.

De los 38 niños y adolescentes tratados con TCC, en seis casos, se añadió a la terapia psicológica tratamiento psicofarmacológico. Para ello, se emplearon distintos grupos de fármacos, solos o combinados: benzodiacepinas (Iorazepam o alprazolam), antidepresivos tricíclicos (imipramina, mirtazapina o clomipramina) e inhibidores selectivos de la recaptación de serotonina (ISRS) (fluoxetina o paroxetina).

La duración media del tratamiento con TCC o combinado con fármacos es de 4,1 meses (DE: 9,3), con resultados divergentes en los distintos estudios: 32 recuperaciones completas ( $80 \%$ ), seis recuperaciones parciales (14,2\%) (ingestas adecuadas con 
selección alimentaria mantenida), dos transformaciones (virado de la sintomatología hacia otro trastorno del mismo espectro), una pérdida de seguimiento del caso y una recuperación completa con recidiva posterior.

La inclusión del tratamiento farmacológico no incorpora ventajas en el tiempo de resolución del problema, aunque tenga una incidencia directa en la expresión de ansiedad. Al tratarse de una muestra poco homogénea en cuanto al tiempo de tratamiento, podemos señalar que el $75 \%$ muestran un período terapéutico superior en los casos tratados con fármacos o terapia combinada (6,5 y 5 meses, respectivamente) frente a los 3,25 meses de la TCC, aunque desconocemos el grado de gravedad de cada uno de estos casos.

La gran variabilidad de la muestra es debida a la distinta frecuencia de tratamiento en los casos y los criterios de remisión completa que se emplean. Así, Burklow y Linscheid ${ }^{12}$ emplean el ingreso en hospitalización para el tratamiento de los casos, encontrando una mejoría en la sintomatología en pocos días, y remisión total del cuadro en pocas semanas. En cambio, Okada et al. ${ }^{4}$, en su revisión de seis casos tratados con TCC, cifran la recuperación total de dos de sus pacientes atendidos en 18 y 48 meses después del inicio del tratamiento ambulatorio del trastorno.

En 2005, Banerjee et al. ${ }^{20}$ publican tres casos de fobia a la deglución refractarios al tratamiento con TCC, con grave comorbilidad con TOC y trastorno de ansiedad no especificado, a los que se les aplica tratamiento con ISRS sin presencia de TCC, con resultados satisfactorios y recuperación completa del trastorno. La duración media del tratamiento farmacológico hasta la remisión completa es de 7,5 meses (intervalo de 2 a 9 meses).

Es importante remarcar una única investigación publicada que nos permite obtener datos del tratamiento con desensibilización y reprocesamiento por movimientos oculares (EMDR) en la fobia a la deglución ${ }^{9}$. Los cuatro casos analizados obtienen resultados de remisión completa del trastorno en una o dos sesiones, aunque los propios autores señalan que
La enfermera puede realizar una aportación importante al proceso de valoración y tratamiento en los diversos niveles de atención

se precisaron diversas sesiones más para ofrecer tratamiento dirigido al tipo de vínculo y dificultades familiares que se habían creado por la evolución del trastorno para facilitar la recuperación total del problema. Estos casos, aparentemente, estaban exentos de comorbilidad con otros trastornos. El tratamiento se basa en diversos pasos que se adjuntan a las ocho fases del protocolo estándar de EMDR: a) desensibilización de los recuerdos de destino (desensibilización del primer recuerdo, del recuerdo más doloroso y de la experiencia más reciente, en tres fases); b) plantilla de futuro (exposición en imaginación); c) vídeo mental de comprobación de la situación fóbica; d) exposición en vivo y experimentos conductuales. Los resultados son muy esperanzadores, pero, en la publicación, se señalan las limitaciones propias del estudio: inexistencia de medidas estandarizadas, escaso número de casos y sin comorbilidad con otros trastornos, que son insuficientes para extraer conclusiones.

El tiempo de seguimiento de los pacientes no sigue un patrón estándar en todos los casos estudiados, con gran variabilidad en las visitas de control (desde un mes a un año) para establecer una recuperación completa de la fobia específica.

\section{Enfermería en el proceso terapéutico}

En la mayoría de las publicaciones, no se menciona el papel fundamental que posee el equipo multidisciplinario en el tratamiento de las fobias específicas. En un artículo reciente de Lopes et al..21, se hace énfasis en la necesidad de la aproximación multidisciplinaria de estos trastornos. El papel que la enfermera de los equipos de salud mental infantojuvenil puede desempeñar no se refleja en la mayoría de publica- 
ciones, incluso, en aquellas en que el tratamiento se realiza en las unidades de hospitalización. Únicamente en un artículo centrado en los cuidados que realizamos los autores de este estudio de revisión ${ }^{22}$, se explica de forma pormenorizada el papel que puede desempeñar una enfermera especialista en salud mental en el plan terapéutico de la fobia a tragar.

\section{DISCUSIÓN}

La baja incidencia del trastorno en la edad infantil y la escasa importancia que se le reconoce repercute en el reducido número de publicaciones sobre el problema y en que no dispongamos hasta el momento de un tratamiento psicológico o farmacológico que reúna las garantías metodológicas para considerarse bien establecido. El trastorno tiene mayor prevalencia en el sexo femenino en la etapa infantil y juvenil, aumentando la proporción en la etapa adulta14.

Aunque los diversos autores citados señalan claramente la gravedad de algunos cuadros, con afectación importante de diversas esferas del niño, sigue considerándose un trastorno menor. La aparición de la fobia a tragar en la primera infancia disminuye el tiempo de evolución del problema, comparado con los casos que se citan en la literatura de personas adultas, pero es llamativo el tiempo de evolución de algunos casos citados, en los que ni los cuidadores principales ni los servicios sanitarios preventivos han detectado y tratado estas deficiencias alimentarias durante años.

Hay poca homogeneidad, incluso en los artículos más recientes, en el análisis de los casos y sus posibles causas y efectos, aunque, en las distintas publicaciones, se han ido incorporando diversos factores de riesgo, mantenimiento y protección de la fobia a la deglución. En próximas publicaciones, se deberían incluir los diferentes factores en el análisis de los casos, que otros autores han introducido en sus artículos, con la finalidad de poder esclarecer su importancia en el desarrollo y la evolución del trastorno.
El término consensuado entre los distintos artículos consultados ha sido el diagnóstico diferencial: la mayoría de autores está de acuerdo en que ha de incluirse la fobia a la deglución en el diagnóstico inicial de diversos cuadros, inicialmente catalogados de trastornos alimentarios, y nos facilitan elementos clave para diferenciarla de las distintas enfermedades con sintomatología similar.

La evolución del cuadro suele ser favorable, siendo mejor cuanto más precozmente se instaure el tratamiento. Este es básicamente psicológico, incluyendo, en los casos graves, separación del medio familiar por la sobreprotección y ansiedad que genera, con el ingreso hospitalario de los niños.

El principal tratamiento publicado en la fobia a la deglución es la TCC, con amplia diversidad en las técnicas y resultados obtenidos en la práctica clínica. Parece que su asociación a fármacos (principalmente, antidepresivos y benzodiacepinas) no facilita la reducción del tiempo de tratamiento. Podría ser que propiciara otros aspectos del tratamiento que no han sido considerados en las diferentes publicaciones. La unificación de un protocolo de tratamiento conferiría homogeneidad al proceso y facilitaría su posterior análisis.

La EMDR es una terapia con resultados prometedores, pero que requiere de mayor difusión y utilización en la práctica asistencial en este trastorno a fin de poder generalizar los resultados obtenidos.

Para la valoración global del trastorno, es necesaria una visión holística de la afectación en todas las áreas del niño y de la familia, que incluya: gravedad del trastorno, tiempo de evolución, posibles desencadenantes, factores mantenedores, diagnósticos comórbidos o sintomatología predominante, interacción parental y con iguales, protocolo terapéutico y resultado del seguimiento a largo plazo. Para ello, es necesario un abordaje multidisciplinario, que no se contempla en las diversas publicaciones presentadas. La enfermera, dentro de los equipos de atención de salud mental infantojuveniles, puede realizar una aportación importante al proceso de valoración y tratamiento en sus diversas apro- 
ximaciones: detección de los factores de riesgo, identificación de signos de alarma, valoración inicial de la gravedad del cuadro, psicoeducación a la familia, intervención directa en el proceso terapéutico mediante el entrenamiento en técnicas de relajación hacia el niño, ejecución de las exposiciones en imaginación y en vivo, y establecimiento de un vínculo positivo con el chico y la familia que facilite el cumplimiento de las pautas fijadas, el aumento de la motivación y los sentimientos de autoeficacia, favoreciendo la expresión de los sentimientos, dudas y temores que puedan surgir a lo largo de la fase terapéutica.

Es necesario el conocimiento extenso de este cuadro por parte de las enfermeras de asistencia primaria, incluyendo su detección en el programa de control del niño sano, como ya se hace con otros trastornos de la alimentación, con la finalidad de instaurar una terapia adecuada lo más tempranamente posible dirigida al núcleo familiar, debido a la buena respuesta que los niños y adolescentes suelen tener y al aumento de frecuencia de la enfermedad.

\section{BIBLIOGRAFÍA}

1. López-Guimerà G, Sánchez-Carracedo D. Prevención de las alteraciones alimentarias: fundamentos teóricos y recursos prácticos. Madrid: Pirámide; 2010.

2. Kaplan PR, Evans IM. A case of functional dysphagia treated on the model of fear of fear. J Behav Ther Exp Psychiatry. 1978;9(1):71-2.

3. LeBeau RT, Glenn D, Liao B, Wittchen HU, BeesdoBaum K, Ollendick T, et al. Specific phobia: a review of DSM-IV specific phobia and preliminary recommendations for DSM-V. Depress Anxiety. 2010;27(2):148-67.

4. Okada A, Tsukamoto C, Hosogi M, Yamanaka E, Watanabe K, Ootyou K, et al. A study of psycho-pathology and treatment of children with phagophobia. Acta Med Okayama. 2007;61(5):261-9.

5. López Ibor JJ, Valdés Miyar M. DSM-IV-TR: manual diagnóstico y estadístico de los trastornos mentales: texto revisado. Barcelona: Masson; 2002.

6. American Psychiatric Association. DSM-5. Manual diagnóstico y estadístico de los trastornos mentales. Madrid: Editorial Médica Panamericana; 2014.

7. Bailly D, de Chouly de Lenclave MB, Dhaussy S, Baert
F, Turck D. La phobie de déglutition chez l'enfant : un diagnostic différentiel de l'anorexie mentale. Arch Pediatr. 2003;10(4):337-9.

8. Çiyiltepe M, Türkbay T. Phagophobia: a case report. Turk J Pediatr. 2006;48(1):80-4.

9. de Roos C, de Jongh A. EMDR treatment of children and adolescents with a choking phobia. J EMDR Pract Res. 2008;2(3):201-11.

10. Montañés-Rada F, de Lucas-Taracena MT, MartínGranero MA, Sánchez-Romero S. Fobia a tragar: presentación clínica de una serie de nueve casos. An Psiquiatr. 2005;21(6):289-96.

11. Zelikovsky N, MacNaughton KL, Geffken GR. A review of choking phobia in children: diagnosis, assessment, and treatment. J Psychol Pract. 2001;7(1):23-32.

12. Burklow KA, Linscheid T. Rapid inpatient behavioral treatment for choking phobia in children. Child Health Care. 2004;33(2):93-107.

13. de Lucas-Taracena MT, Ibarra I. Swallowing phobia: a case report. Actas Esp Psiquiatr. 2001;29(6):411-3.

14. de Lucas-Taracena MT, Montañés-Rada F. Fobia a atragantarse en la infancia y adolescencia. Rev Psiquiatr Infanto-Juvenil. 2005;22(3):92-101.

15. Chorpita BF, Vitali AE, Barlow DH. Behavioral treatment of choking phobia in an adolescent: an experimental analysis. J Behav Ther Exp Psychiatry. 1997; 28(4):307-15.

16. Gómez-Mayorga ME, del Barrio-Gándara MV. Tratamiento de la fobia a tragar: estudio de un caso. Acción Psicológica. 2002;1(3):273-84.

17. Geffken G, Sajid M, MacNaughton K. The course of childhood OCD, its antecedents, onset, comorbidities, remission, and reemergence: a 12-year case report. Clin Case Stud. 2005;4(4):380-94.

18. García-de Ribera C, Marugán-de Miguelsanz JM, Geijo-Uribe S, Imaz-Roncero C. Miedo al atragantamiento: ¿trastorno alimentario o fobia específica? Bol Pediatr. 2010;50(211):25-7.

19. Tanıdır C, Hergüner S. Mirtazapine for choking phobia: report of a pediatric case. J Child Adolesc Psychopharmacol. 2015;25(8):659-60.

20. Banerjee SP, Bhandari RP, Rosenberg DR. Use of lowdose selective serotonin reuptake inhibitors for severe, refractory choking phobia in childhood. J Dev Behav Pediatr. 2005;26(2):123-7.

21. Lopes R, Melo R, Curral R, Coelho R, Roma-Torres A. A case of choking phobia: towards a conceptual approach. Eat Weight Disord. 2014;19(1):125-31.

22. Torralbas-Ortega J, Puntí-Vidal J, Valls-Ibáñez MV, Arias-Núñez E, Naranjo-Díaz MC. Fobia a tragar, plan de cuidados en la infancia y adolescencia. Enferm Clin. 2012;22(4):224-30. 